\title{
Increased mitochondrial DNA copy number protects hair cells and HEI-OC1 cells against drug-induced apoptosis
}

\author{
HONGLIN MEI $^{1 *}$, DONGMEI MEI ${ }^{2 *}$, HUIQIAN YU $^{1 *}$, SHAN SUN $^{1}$, YAN CHEN $^{1}$, \\ YANPING ZHANG ${ }^{1}$, RENJIE CHAI ${ }^{3}$ and HUAWEI LI ${ }^{1}$
}

\begin{abstract}
${ }^{1}$ ENT Institute and Department of Otorhinolaryngology, Affiliated Eye and ENT Hospital, State Key Laboratory of Medical Neurobiology, Fudan University, Shanghai 200031; ${ }^{2}$ Department of Stomatology, Key Laboratory of Oral Clinical Medicine, The Affiliated Hospital of Qingdao University, College of Stomatology, Qingdao University, Qingdao, Shandong 266000; ${ }^{3}$ Co-Innovation Center of Neuroregeneration, Key Laboratory for Developmental Genes and Human Disease, Ministry of Education, Institute of Life Sciences, Southeast University, Nanjing, Jiangsu 210096, P.R. China
\end{abstract}

Received June 7, 2019; Accepted October 23, 2019

DOI: $10.3892 / \mathrm{mmr} .2019 .10838$

\begin{abstract}
Several factors trigger apoptosis in cochlear hair cells. Previous studies have shown that mitochondria play key roles in apoptosis, but the role of mitochondrial deoxyribonucleic acid (mtDNA) copy number in the pathogenesis of hair cell apoptosis remains largely unknown. We used mouse cochlear hair cells and House Ear Institute-Organ of Corti 1 (HEI-OC1) cells to explore the relationship between mtDNA copy number and cell apoptosis. We found that the mtDNA copy number of hair cells was reduced relative to mitochondrial mass and hypothesized that increasing it might have a protective effect. We then increased the mtDNA copy number of the hair and HEI-OC1 cells by transfecting them with an adeno-associated virus (AAV) vector containing mitochondrial transcription factor A (TFAM). We found that the apoptosis rates decreased upon inducing apoptosis with
\end{abstract}

Correspondence to: Dr Huawei Li or Dr Shan Sun, ENT Institute and Department of Otorhinolaryngology, Affiliated Eye and ENT Hospital, State Key Laboratory of Medical Neurobiology, Fudan University, 83 Fenyang Road, Shanghai 200031, P.R. China

E-mail: hwli@shmu.edu.cn

E-mail: shansun@fudan.edu.cn

${ }^{*}$ Contributed equally

Abbreviations: DDP, cisplatin; HEI-OC1, House Ear Institute-Organ of Corti 1; mtDNA, mitochondrial deoxyribonucleic acid; mPTP, mitochondrial permeability transition pore; ROS, reactive oxygen species; TFAM, mitochondrial transcription factor A; MMP, mitochondrial membrane potential; FCM, flow cytometry; TUNEL, TdT-mediated dUTP nick-end labeling; FITC, fluorescein isothiocyanate; PI, propidium iodide

Key words: apoptosis, cochlear hair cells, HEI-OC1, mitochondrial DNA copy number, mitochondrial permeability neomycin or cisplatin (DDP). To elucidate the mechanisms, we analyzed the mitochondrial-membrane permeability and mitochondrial function of HEI-OC1 cells. Our results suggested that the increase in mtDNA copy number could protect hair cells and HEI-OC1 cells against drug-induced apoptosis by stabilizing the permeability of the mitochondrial membrane and mitochondrial function.

\section{Introduction}

Disabling hearing loss affects more than $5 \%$ of the world's population (466 million individuals). It is estimated that by 2050 , more than 900 million people will be affected by disabling hearing loss (http://www.who.int/en/news-room/fact-sheets/detail/deafness-and-hearing-loss). Sensorineural hearing loss (SNHL) is a common disease worldwide that accounts for most hearing loss. Currently, there are no effective treatment options available. Factors that may lead to SNHL include aging, exposure to loud noise, heredity, and some medications (1). All of these factors may result in the apoptosis or loss of cochlear hair cells. Because hair cells cannot regenerate once they are lost $(2,3)$, how to protect them against apoptosis, either by inhibiting processes that lead to damage or by enhancing their survival, is obviously an important research goal.

The mitochondrion plays an important role in cell apoptosis; it is also the main site of intracellular oxidative phosphorylation and adenosine triphosphate (ATP) synthesis (4). Mitochondria have their own nucleic acid, mitochondrial deoxyribonucleic acid (mtDNA). As reported $(5,6)$ a variation in the mtDNA copy number can affect cell apoptosis. Transgenic mice with mtDNA deletions cannot survive the embryonic period, and a large number of apoptotic cells have been found in such embryos (5). Our previous research also found that decreased mtDNA copy number sensitizes cancer cells to chemotherapeutic drugs (6). However, its effect on hair cells has not been elucidated. Increased mtDNA copy number may enhance the survival of hair cells and protect them against apoptosis. In the present study, the mtDNA copy number of mouse cochlear 
hair cells was assessed and it was found that normal cochlear hair cells were relatively lacking in mtDNA. Then the mtDNA copy number of hair cells and House Ear Institute-Organ of Corti 1 (HEI-OC1) cells was increased by mitochondrial transcription factor A $(T F A M)$ transfection to ascertain whether it had a protective effect. Finally, the mechanisms by which increased mtDNA copy number protects against apoptosis were explored.

\section{Materials and methods}

Cochlear basal membrane and HEI-OCl cell culture. A total of 50 newborn C57BL/6J mice (P1-P3), each weighing 1.5-2.5 grams, were used in this experiment, and their gender selection was random. International Council for Laboratory Animal Science (ICLAS) Ethical Guideline (https://iclas. org/guidelines-for-researchers) and the ethical requirements of experimental animals in Fudan University (http://labanimal. fudan.edu.cn/95/6f/c5213a38255/page.htm) were followed. Our laboratory animals were cared for by a full-time breeder; our experiment was approved by the Animal Care and Use Committee of the Affiliated Eye and ENT Hospital, Fudan University (no. 201526). Mouse pups were decapitated and dissections were performed under a light microscope in a Petri dish containing ice-cold phosphate-buffered saline (PBS). The cochleae were removed from the skulls and opened with forceps. The basal membranes were detached and transferred into pre-prepared culture dishes containing Dulbecco's modified Eagle's medium-Ham's F-12 (DMEM/F12; Thermo Fisher Scientific, Inc.) supplemented with N2 and B27 (Thermo Fisher Scientific, Inc.), and cultured at $37^{\circ} \mathrm{C}$ with $5 \% \mathrm{CO}_{2}$. To induce apoptosis, neomycin (Sigma-Aldrich; Merck KGaA) was added at a final concentration of $1 \mathrm{mM}$, and then a terminal deoxynucleotidyl transferase (TdT)-mediated dUTP nick end labeling (TUNEL) kit (Thermo Fisher Scientific, Inc.) was utilized to mark the apoptotic cells.

HEI-OC1 is a widely used progenitor hair cell line derived from mouse auditory organs (7). The HEI-OC1 cells were grown in DMEM (Gibco Laboratories; Thermo Fisher Scientific, Inc.) containing $10 \%$ fetal bovine serum (Gibco Laboratories; Thermo Fisher Scientific, Inc.) at $33^{\circ} \mathrm{C}$ with $10 \% \mathrm{CO}_{2}$ and subcultured at $80 \%$ confluence using $0.25 \%$ trypsin/ethylenediaminetetraacetic acid (EDTA; Thermo Fisher Scientific, Inc.). To induce apoptosis, cisplatin (DDP; Sigma-Aldrich; Merck KGaA) was added at a final concentration of $0.07 \mathrm{mM}$.

Measurement of mtDNA copy number and transcription of mtDNA genes using real-time quantitative polymerase chain reaction $(q P C R)$. Total RNA and DNA were extracted with a DNA/RNA Isolation Kit (Qiagen). Reverse transcription was performed using a PrimeScript RT Reagent Kit with gDNA Eraser (Takara) per the manufacturer's protocols. The mitochondrial gene NDl was used to represent mtDNA copy number. The relative mtDNA copy number was defined as the total amount of mtDNA divided by the total amount of nuclear DNA.

qPCR was performed using the Applied Biosystems 7500 Real-Time PCR System (Thermo Fisher Scientific, Inc.) using GoTaq qPCR Master Mix (Promega). We designed validated primers for each target messenger RNA (mRNA) or DNA:
TFAM forward, ATGGCGTTTCTCCGAAGCAT and reverse, CAGATGAAAACCACCTCGGTAA; COI forward, ACTCCT ACCACCATCATTTCTCC and reverse, GGCTAGATTTCC GGCTAGAGG; COII forward, CTTGGTCTACAAGACGCC $\mathrm{AC}$ and reverse, CTATTGGCAGAACGACTCGG; $12 \mathrm{SrRNA}$ forward, AATGAAGTACGCACACACCG and reverse, GGG TGTAGGCCAGATGCTTT; ATP6 forward, AAGCTCACT TGCCCACTTCC and reverse. GTAAGCCGGACTGCTAAT GC; GAPDH forward, TGCGACTTCAACAGCAACTC and reverse, CTTGCTCAGTGTCCTTGCTG; NDl forward, ACC ATTTGCAGACGCCATAA and reverse, TGAAATTGTTTG GGCTACGG; ACTB forward, TCCCAGTTGGTAACAATG CCA and reverse, TGTTCCCTTCCACAGGGTGT. qPCR conditions consisted of an initial denaturing step of $30 \mathrm{sec}$ at $95^{\circ} \mathrm{C}$ followed by 35-40 cycles of $5 \mathrm{sec}$ of denaturation at $95^{\circ} \mathrm{C}, 20 \mathrm{sec}$ of annealing at $60^{\circ} \mathrm{C}$ and $20 \mathrm{sec}$ of extension at $72^{\circ} \mathrm{C}$. The results were calculated using the comparative-cycle threshold $(\Delta \Delta \mathrm{Cq})$ method (8).

Adeno-associated virus (AAV) transfection. An AAV vector containing human TFAM (Hanbio) was used in this experiment. The control encoded a scrambled 150-bp nucleotide sequence and expressed green fluorescent protein (GFP) (Hanbio). When cochlear membranes had been cultured for $24 \mathrm{~h}$ or HEI-OC1 cells cultivated to $80 \%$ confluence, $4 \mu \mathrm{l}$ AAV-TFAM or the control $\left[2 \times 10^{12}\right.$ viral genomes per $\mathrm{ml}$ $(\mathrm{vg} / \mathrm{ml})]$ was added into $1 \mathrm{ml}$ culture medium for $24 \mathrm{~h}$, and tissues or cells were collected at the appropriate time points and then fixed in $4 \%$ paraformaldehyde.

Fluorescence in situ hybridization (FISH). The probe was prepared using the FISH Tag DNA Kit (Thermo Fisher Scientific, Inc.), and the spectrum of the intended probe was from 278 to 1435 bases (NCBI Reference Sequence: NC_005089.1) in mouse mtDNA.

The cochlear basal membranes or HEI-OC1 cells were cultured, collected at proper time points on slides, and then fixed with $4 \%$ polyoxymethylene and permeabilized with $0.5 \%$ Triton X-100. Then, the FISH procedure was conducted as previously described (6).

Measurement of mitochondrial mass and immunofluorescence. MitoTracker Green FM (Thermo Fisher Scientific, Inc.) was used to determine mitochondrial mass, and MitoSOX Red (Thermo Fisher Scientific, Inc.) was used to determine reactive oxygen species (ROS) levels. Cochlear basal membranes were grown in culture medium for a certain period of time, the culture medium was removed and the samples were washed with PBS. We then added a pre-warmed $\left(37^{\circ} \mathrm{C}\right)$ solution containing MitoTracker Green FM or MitoSOX Red and the cells were incubated at $37^{\circ} \mathrm{C}$ for $20 \mathrm{~min}$. After staining, the samples were washed in PBS and imaged (living-cell imaging) under a Leica SP8 confocal microscope (magnification, $x 40$; Leica Biosystems). To label hair cells, anti-myosin VIIA rabbit polyclonal antibody (ab3481, Abcam) was added and the cells were incubated for $8 \mathrm{~h}\left(4^{\circ} \mathrm{C}\right)$ at a dilution of 1:200 after cochlear basal membranes had been fixed with $4 \%$ polyoxymethylene and permeabilized with $0.5 \%$ Triton X-100. We washed the samples 3 times with PBS and incubated them for $2 \mathrm{~h}$ with fluorescence-conjugated goat anti-rabbit immunoglobulin $\mathrm{G}$ 
(IgG) secondary antibody (ab150077, Abcam) at room temperature (RT).

Since GFP expression was extremely low on the second day after TFAM transfection and signals could not be detected after FISH treatment, we used anti-GFP (ab290, Abcam) to enhance the signals. The immunofluorescence procedure was the same as mentioned above.

Flow cytometry (FCM). To measure mitochondrial permeability, we conducted FCM using a MitoProbe Transition Pore Assay kit (M34153, Thermo Fisher Scientific, Inc.) according to the manufacturer's protocols. For each sample, we prepared 3 aliquots: 1 contained calcein-acetoxymethyl (calcein AM) only; 1 contained calcein $\mathrm{AM}$ and $\mathrm{CoCl}_{2}$; and 1 contained calcein $\mathrm{AM}, \mathrm{CoCl}_{2}$ and ionomycin. The samples were incubated at $37^{\circ} \mathrm{C}$ in the dark for $15 \mathrm{~min}$. Detailed information can be found in the manufacturer's protocols.

To determine mitochondrial membrane potential (MMP) and analyze reactive oxygen species (ROS), the HEI-OC1 cells were cultured, trypsinized and collected; and then resuspended in a pre-warmed $\left(37^{\circ} \mathrm{C}\right)$ solution containing MitoTracker Red CMXRos or MitoSOX Red (Thermo Fisher Scientific, Inc.) for $10 \mathrm{~min}$. The HEI-OC1 cells were then washed with PBS; and they were analyzed by FCM. For apoptosis analysis, a TUNEL assay kit was utilized. First, the cells were collected and fixed with paraformaldehyde, and then centrifuged for $5 \mathrm{~min}$ at $300 \mathrm{xg}$ and then the supernatant was discarded. Next, the cells were washed in PBS, pelleted by centrifugation and then added to $5 \mathrm{ml}$ ice-cold $70 \%$ (v/v) ethanol. Finally, the cells were analyzed by FCM. A Dead Cell Apoptosis Kit with Annexin V-FITC and PI (Thermo Fisher Scientific, Inc.) was used. The cells were washed twice with cold PBS and then resuspended at a concentration of $1 \times 10^{6}$ cells $/ \mathrm{ml}$. We added Annexin V/PI, gently mixed it with the cells, incubated the mixture at RT in the dark and then analyzed the cells by FCM as soon as possible. All tests were repeated at least 3 times.

Statistical analysis. For all experiments, values for the normal controls were set to 1, except for the experiments of flow cytometry. Therefore, most of our histograms show relatively quantified (RQ) values. We made cross-sectional reconstruction after confocal microscope scanning in all our hair cell imaging except for the apoptosis experiments. Al fluorescence quantification was performed with Adobe Photoshop CC (Adobe Systems Inc.). The targeted area (hair cell) was selected and the average brightness of each cell in gray mode was calculated. At least 50 cells in each group were selected. We repeated this procedure at least 3 times for all our experiments. Statistical analyses were conducted with SigmaPlot software version 12.3 (Systat Software, Inc.). A 2-tailed $\mathrm{t}$ test was used to compare values between groups. Data are presented as mean \pm standard deviation (SD). The difference was considered significant when $\mathrm{P}<0.05$.

\section{Results}

Relative deficiency of mtDNA copy number and high levels of ROS in hair cells. Mitochondria are the powerhouses of cells. As auditory receptors and mechano-electrical transducers, hair cells need a large number of mitochondria, which we confirmed in this experiment. After culturing mouse cochlear basal membranes in vitro for $24 \mathrm{~h}$, we measured their mitochondrial mass using MitoTracker Green, for which the location in mitochondria is not affected by MMP. We observed that the fluorescence of hair cells was extremely intense compared with that of supporting cells, indicating that the mitochondrial mass of hair cells was significantly higher (Fig. 1A and D; $t=14.024 ; \mathrm{n}=5 ; \mathrm{P}=0.000000649$ ). We also performed FISH on mtDNA in cochlear basal membranes after culturing them for $24 \mathrm{~h}$ in vitro. In this experiment, we found that the mtDNA copy number of hair cells was also higher than that of supporting cells (Fig. 1B and $\mathrm{E} ; t=4.083 ; \mathrm{n}=6 ; \mathrm{P}=0.00274$ ), but the difference in fluorescence intensity between hair cells and supporting cells was smaller than the difference in mitochondrial mass between both types of cells. This demonstrated that the copy number per mitochondrion was relatively deficient in hair cells compared with that of supporting cells (Fig. 1G; $t=9.366 ; n=5 ; \mathrm{P}=0.0000138$ ), which may have influenced normal mitochondrial function. Finally, we measured the ROS level using MitoSOX Red and observed that it was higher in hair cells than that in supporting cells (Fig. 1C and F; $t=4.6422 ; \mathrm{n}=5 ; \mathrm{P}=0.00166$ ), which may have been caused in part by relative mtDNA copy number deficiency.

Protective effect of increased mitochondrial DNA copy number on hair and HEI-OCl cells. TFAM is an important gene that regulates the copy number of mitochondrial DNA, and its overexpression has no effect on mitochondrial mass (9). We used AAV-TFAM GFP to increase hair cell mtDNA copy number in this experiment (see our 'Materials and methods' section for detailed information). After culturing mouse cochlear basal membranes in vitro for $24 \mathrm{~h}, \mathrm{AAV}-T F A M$ was added into the medium, and FISH was performed on mtDNA and qPCR on TFAM RNA. It was found that the mtDNA copy number was significantly increased in some hair cells at the second day after transfection (Fig. 2A, B and E; $t=4.44 ; n=9$; $\mathrm{P}=0.000412$ ). The result was confirmed in our qPCR experiment (Fig. 2F; $t=5.052 ; \mathrm{n}=3 ; \mathrm{P}=0.00722$ ). We also found that mRNA levels of TFAM were significantly increased (Fig. 3A; $t=13.698,19.14,17.638 ; \mathrm{n}=3,3,3 ; \mathrm{P}=0.000165,0.0000439$, $0.0000607)$. At the fifth day after transfection, it was found that the GFP signal remained in some hair cells, but the mtDNA copy number of transfected hair cells was no longer higher than the non-transfected cells (Fig. 3B-D). The results indicated that the increase in mtDNA copy number occurred only early on after TFAM transfection. As time progressed, TFAM overexpression may have blocked mtDNA replication and offset its effect of increasing mtDNA copy number.

In the apoptosis induction experiment, we added neomycin into the medium at a final concentration of $1 \mathrm{mM}$ on the second day after TFAM transfection. Twelve hours later, we collected the basal membranes and compared hair cell loss or apoptosis rates between the TFAM transfection and control groups using a TUNEL kit. It was demonstrated that the basal membranes of the TFAM transfection group had an obviously lower apoptosis rate than did those of the control group (Fig. 2C, D and G; $t=3.638 ; \mathrm{n}=3, \mathrm{P}=0.022$ ).

To verify the previous results on hair cells, we repeated the experiment on HEI-OC1 cells. After transfecting them with TFAM, we collected the cells at different time points. First, we 

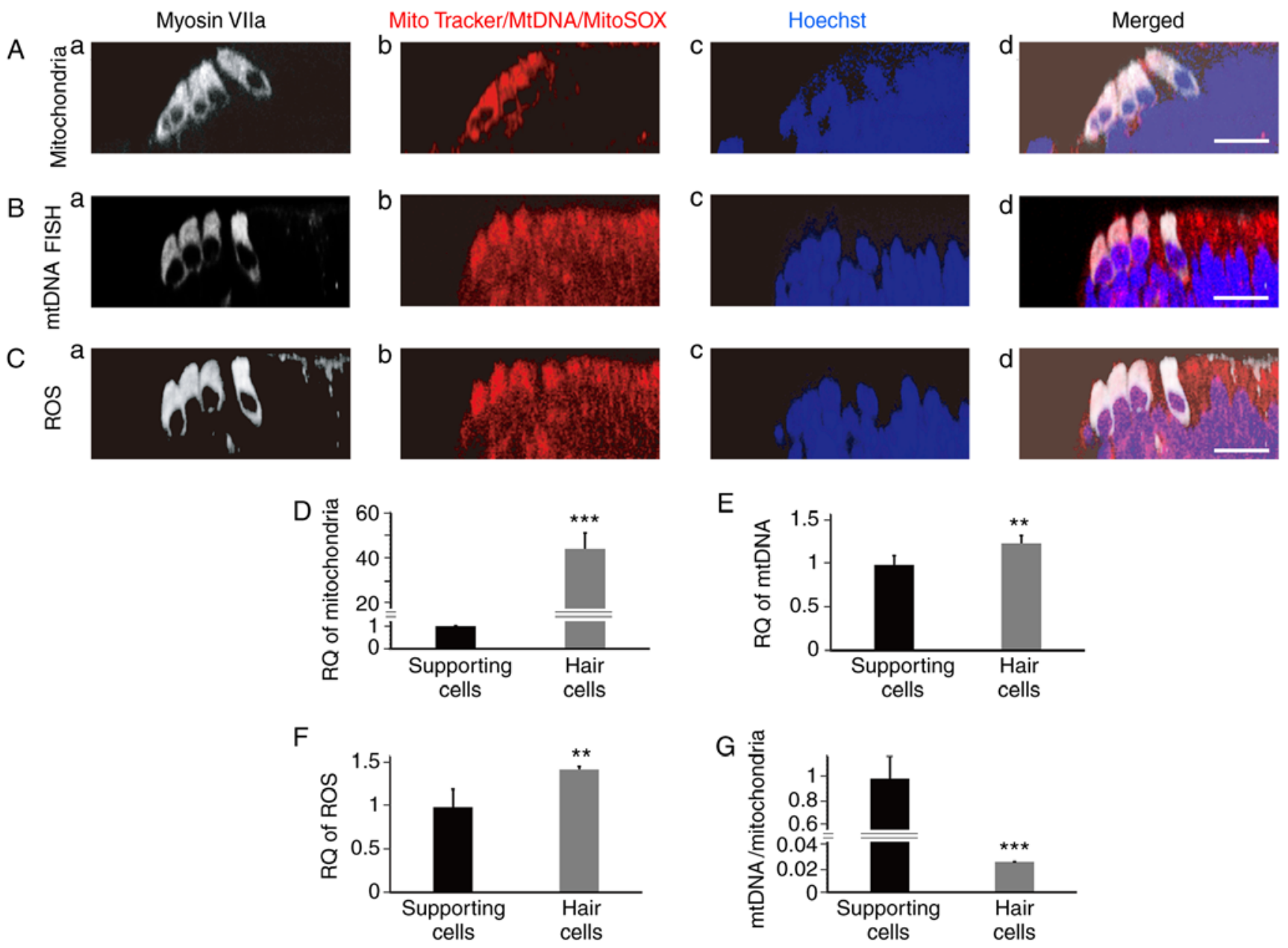

Figure 1. Measurement of mitochondrial mass, mtDNA and ROS in newborn mouse cochlear hair cells. (A) Mitochondria were marked in cochlear basal membranes using MitoTracker Green (cross-sectional reconstruction). The mitochondrial mass of hair cells was significantly higher than that of the supporting cells (a, marked hair cells; b, mitochondrial staining; c, nucleus staining; d, the merged image). (B) FISH of mtDNA in cochlear basal membrane, in which the intensity of red fluorescence is indicative of mtDNA copy number (cross-sectional reconstruction). The mtDNA copy number of hair cells was higher than that of the supporting cells, but the difference in mtDNA copy number between hair cells and supporting cells was obviously smaller than the difference in mitochondrial mass (a, marked hair cells; b, mtDNA staining; c, nucleus staining; d, the merged image). (C) The ROS level was measured using MitoSOX Red (cross-sectional reconstruction). The ROS level in hair cells was higher than that in the supporting cells (a, marked hair cells; b, ROS staining; c, nucleus staining; $d$, the merged image). (D) Quantitative analysis of fluorescence intensity in supporting cells and hair cells in $A(1 \pm 0.198 ; 44.177 \pm 6.881 ; t=14.024 ; \mathrm{n}=5$; $\mathrm{P}=0.000000649)$. (E) Quantitative analysis of fluorescence intensity in supporting cells and hair cells in $\mathrm{B}(1 \pm 0.110 ; 1.252 \pm 0.091 ; t=4.083 ; \mathrm{n}=6$; $\mathrm{P}=0.00274)$. (F) Quantitative analysis of fluorescence intensity in supporting cells and hair cells in $\mathrm{C}(1 \pm 0.210 ; 1.443 \pm 0.040 ; t=4.6422 ; \mathrm{n}=5 ; \mathrm{P}=0.00166)$. $(\mathrm{G}) \mathrm{Quantitative}$ analysis of fluorescence intensity in supporting cells and hair cells in A and B $(1 \pm 0.232 ; 0.027 \pm 0.004 ; t=9.366 ; \mathrm{n}=5 ; \mathrm{P}=0.0000138)$. Fluorescence intensity of mtDNA was divided by that of mitochondrial mass. Scale bars, $20 \mu \mathrm{m} .{ }^{* *} \mathrm{P}<0.01,{ }^{* * * *} \mathrm{P}<0.001$. ROS, reactive oxygen species; RQ, relatively quantified values.

analyzed TFAM RNA expression levels by qPCR and found that the expression had significantly increased, peaking at the second day (Fig. 4A; $t=21.516 ; \mathrm{n}=3 ; \mathrm{P}=0.0000276$ ). Then, we analyzed the change in mtDNA copy number in HEI-OC1 cells by qPCR. The mtDNA copy number was also increased and peaked at the third day, lagging just behind the change in RNA levels (Fig. 4B; $t=6.556 ; \mathrm{n}=3 ; \mathrm{P}=0.0028$ ). However, the mtDNA copy number was obviously decreased after the fifth day but had no statistically significant difference from the control (Fig. 4B). The results were consistent with those we found in hair cells. In the apoptosis induction experiment, the HEI-OC1 cells were treated with $0.07 \mathrm{mM}$ DDP for $24 \mathrm{~h}$, and then the cells were collected and labeled with TUNEL or Annexin V/PI. The apoptosis rates were analyzed by FCM and the results revealed that the rates of the TFAM transfection group were lower (Fig. 4C and D). After quantification of the result, it was found that the difference was statistically significant (Fig. 4E and F; $t=4.797,16.067 ; n=4,3 ; P=0.00301$, 0.0000878).

Increased mtDNA copy number exerts a protective effect by decreasing mitochondrial membrane permeability. In this experiment, the protection mechanisms of mtDNA copy number in HEI-OC1 cells were explored. As previously mentioned, mtDNA copy number peaked at the third day after TFAM transfection, whereupon we induced apoptosis in the HEI-OC1 cells by DDP for $12 \mathrm{~h}$. We then analyzed the mitochondrial permeability of HEI-OC1 cells by FCM using a Mitochondrial Permeability Transition Pore Assay Kit (Fig. 5A) and found that the fluorescence signal was obviously stronger in the TFAM transfection group (Fig. 5B-a). The result were quantified and the difference was found to be statistically significant, indicating that the mitochondrial permeability had decreased (Fig. 5B-b; $t=7.772 ; \mathrm{n}=3 ; \mathrm{P}=0.00148$ ). The increased 

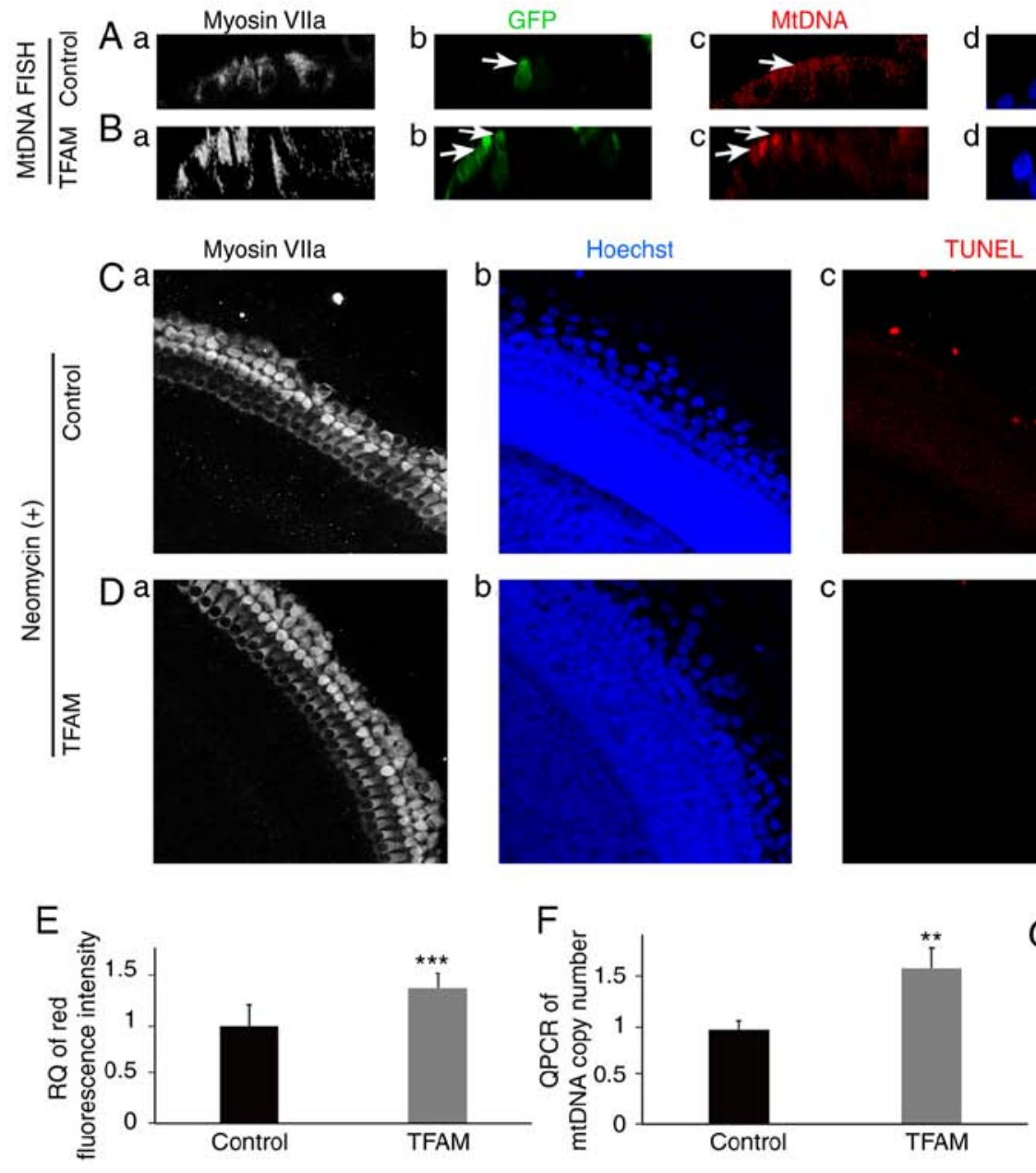

MtDNA
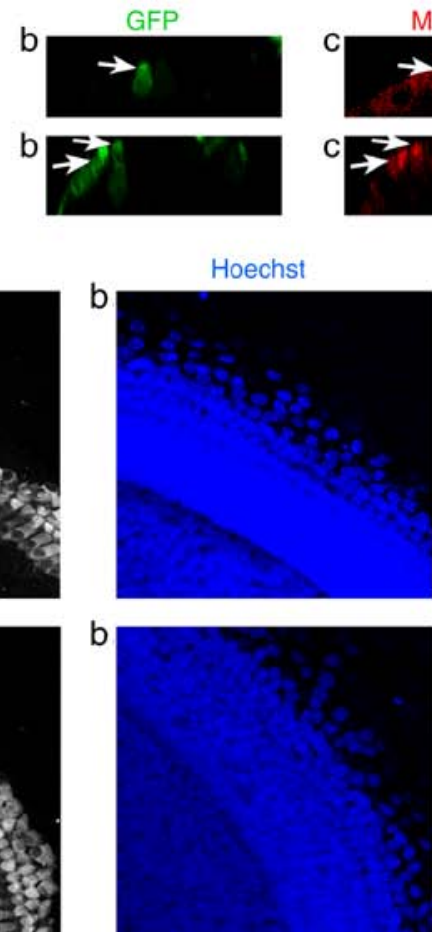

TUNEL
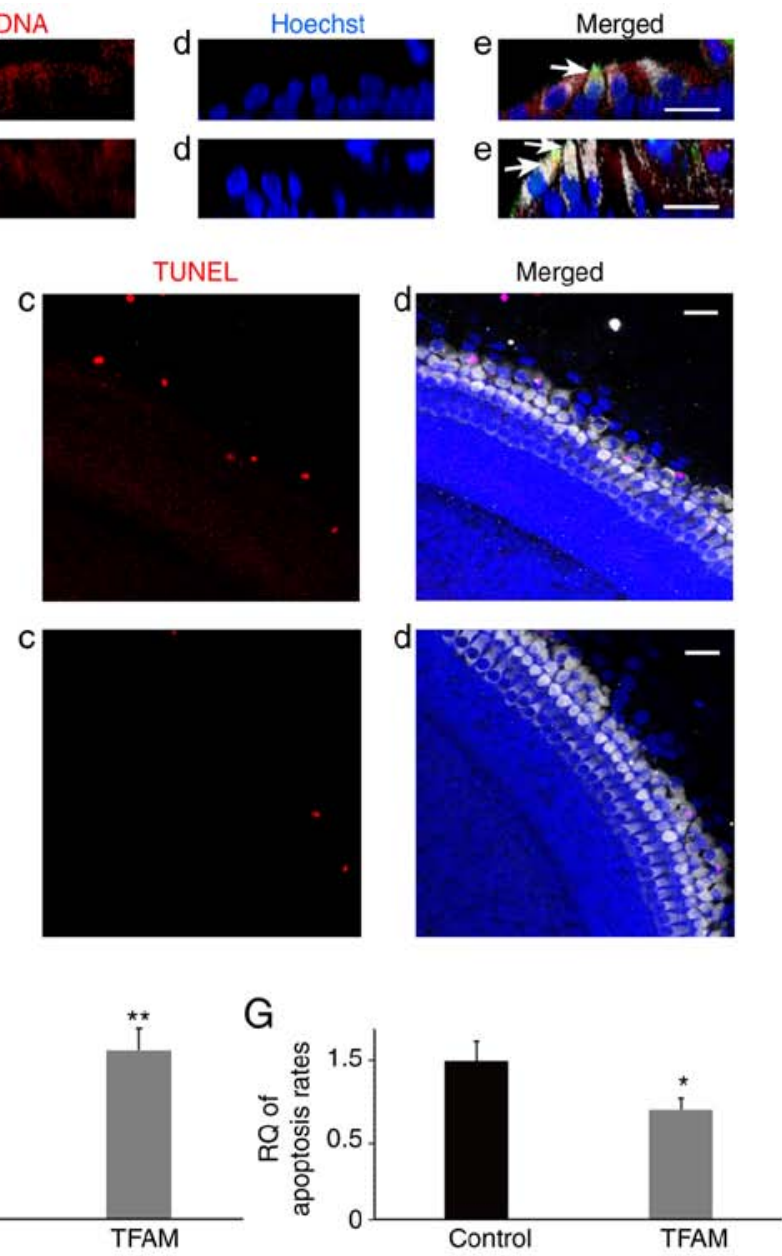

Figure 2. Increased mtDNA copy number has a protective effect on hair cells. (A) FISH of mtDNA in control cochlear basal membrane (a, marked hair cells; $\mathrm{b}$, transfected cells; c, mtDNA staining; $\mathrm{d}$, nucleus staining; e, the merged image), in which the intensity of red fluorescence is indicative of the mtDNA copy number, and the green fluorescence is indicative of the transfected cell (white arrow; cross-sectional reconstruction). (B) FISH of mtDNA in cochlear basal membrane on the second day after TFAM transfection (cross-sectional reconstruction) (a, marked hair cells; b, transfected cells; c, mtDNA staining; d, nucleus staining; e, the merged image). The outer two hair cells were transfected, and the mtDNA copy number was higher (white arrows). (C) We treated the control hair cells with neomycin (a, marked hair cells; b, nucleus staining; c, apoptotic cells; d, the merged image). Many outer hair cells were lost or apoptotic (apoptotic cells are labeled red). (D) We treated hair cells in the TFAM-transfected group with neomycin for the same length of time (a, marked hair cells; b, nucleus staining; c, apoptotic cells; d, the merged image). Few hair cells were lost. (E) Quantitative analysis of red fluorescence intensity in hair cells in A and B $(1 \pm 0.220 ; 1.392 \pm 0.147 ; t=4.44 ; \mathrm{n}=9 ; \mathrm{P}=0.000412)$. (F) qPCR of mtDNA copy number. Cochlear basal membrane mtDNA copy number was increased after TFAM transfection $(1 \pm 0.084 ; 1.649 \pm 0.206 ; t=5.052 ; \mathrm{n}=3 ; \mathrm{P}=0.00722)$. $(\mathrm{G})$ Quantitation of $\mathrm{C}$ and $\mathrm{D}(1 \pm 0.127 ; 0.694 \pm 0.071 ; t=3.638 ; \mathrm{n}=3 ; \mathrm{P}=0.022)$. Apoptosis rate of TFAM-transfected hair cells was lower than that of the control. Scale bars, $20 \mu \mathrm{m} .{ }^{*} \mathrm{P}<0.05,{ }^{* * *} \mathrm{P}<0.01$, ${ }^{* * * *} \mathrm{P}<0.01$. TFAM, mitochondrial transcription factor A; RQ, relatively quantified values.

mtDNA copy number may have blocked the mitochondrial-permeability transition pore (mPTP) when apoptosis was induced in the HEI-OC1 cells. The decrease in mitochondrial permeability may have stabilized mitochondrial function.

Then, we analyzed the mitochondrial transmembrane potential of HEI-OC1 cells by FCM using MitoTracker Red CMXRos at the same time point after DDP treatment. Mitochondrial transmembrane potential was higher in the TFAM transfection group (Fig. 5C-a), but the difference was not statistically significant (Fig. 5C-b). Meanwhile, we analyzed the ROS level of HEI-OC1 cells by FCM using MitoSOX Red. The ROS level was lower in the TFAM transfection group, and the difference was statistically significant (Fig. 5D; $t=9.766 ; \mathrm{n}=3 ; \mathrm{P}=0.000616$ ). After TFAM transfection, increased mtDNA copy number may have blocked the mPTP, further inhibiting the decline of mitochondrial transmembrane potential and suppressing the production of ROS. Ultimately, its protective effect was exerted when apoptosis was induced in the cells.

\section{Discussion}

Mitochondria have their own genetic material, mtDNA, which encodes 13 proteins, 22 transfer RNAs (tRNAs), and 2 ribosomal RNAs (rRNAs), which are involved in maintaining the mitochondrial function. mtDNA copy number varies by cell $(10)$ and by developmental stage $(11,12)$, and depends on the dynamic equilibrium between mtDNA synthesis and degradation. As reported, while a decreased mtDNA copy number or mtDNA depletion may break cellular mitochondrial function and result in many diseases, an increased mtDNA copy number has a protective effect (6,13-15). Given the highly active and vulnerable nature of hair cells, increasing their mtDNA copy number is a potential way to enhance their survival. 

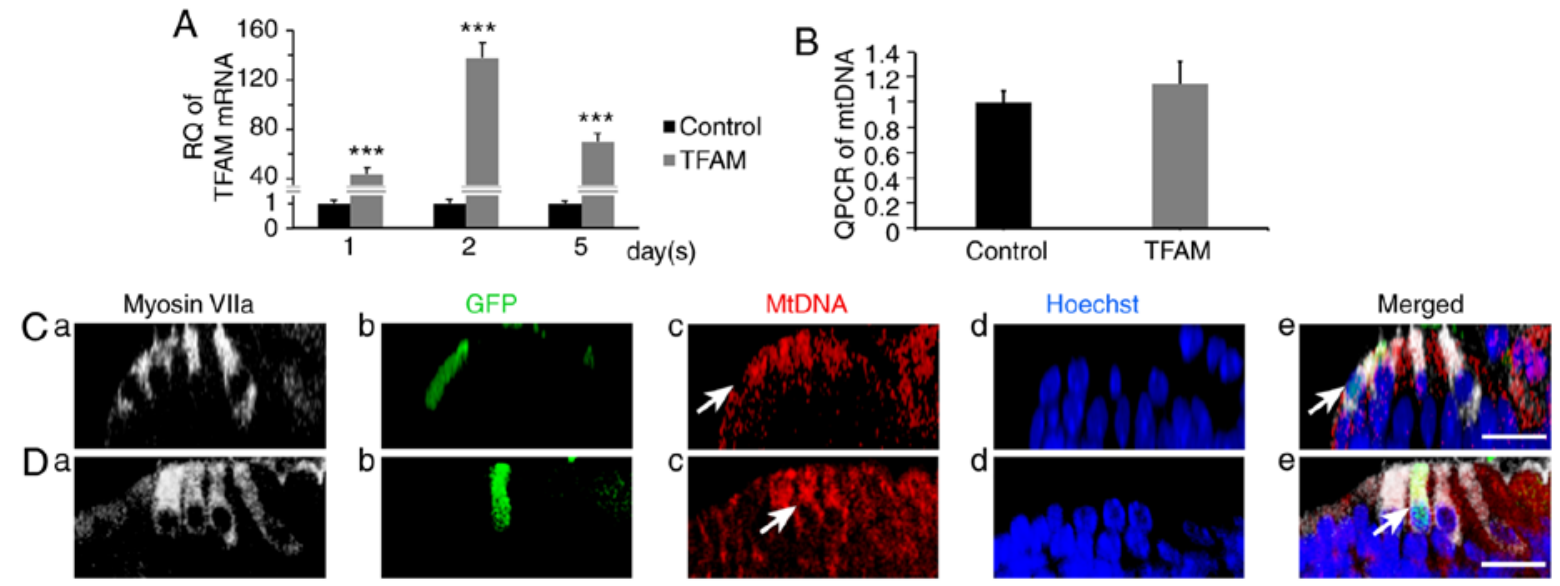

Figure 3. Measurement of the transcription of TFAM and the mtDNA copy number of cochlear hair cells at the fifth day after AAV-TFAM-GFP transfection. (A) qPCR of the mRNA levels of TFAM. At the first, second and fifth day, we extracted the RNA of both control and TFAM-transfected cochlear basal membranes and performed PCR analysis. We found the mRNA levels were statistically higher in the TFAM-transfected group $(1 \pm 0.159 / 43.615 \pm 5.386$, $1 \pm 0.259 / 137.804 \pm 12.377,1 \pm 0.131 / 69.955 \pm 6.770 ; t=13.698,19.14,17.638 ; \mathrm{n}=3,3,3 ; \mathrm{P}=0.000165,0.0000439,0.0000607)$. (B) qPCR of mtDNA copy number. At the fifth day, we extracted the DNA of both control and TFAM-transfected cochlear basal membranes and performed PCR analysis. No statistically significant differences were found $(1 \pm 0.091 ; 1.149 \pm 0.180 ; t=1.282 ; \mathrm{n}=3 ; \mathrm{P}=0.269)$. At the fifth day, the transfected hair cells had bright-green fluorescence, but its mtDNA copy number (red fluorescence) was not higher than those of the other 2 outer hair cells: (C) control group and (D) TFAM group (cross-sectional reconstruction; a, marked hair cells; b, transfected cells; c, mtDNA staining; d, nucleus staining; e, the merged image). Scale bar, $20 \mu \mathrm{m}$. ${ }^{* * *} \mathrm{P}<0.01$. TFAM, mitochondrial transcription factor $\mathrm{A} ; \mathrm{RQ}$, relatively quantified values.

In the present study, we measured the mitochondrial mass, ROS level, and mtDNA copy number in newborn mouse cochlear hair cells. It was found that the mitochondrial mass of hair cells was significantly higher than that of the supporting cells, and the same was true for their ROS level. These results were anticipated as hair cells require a great deal of energy from mitochondria to sustain their normal function, and the levels of by-product ROS increase during the ATP production process. Then, the mtDNA copy number in hair cells was then assessed and it was higher than that of the supporting cells, but to a smaller degree than was true for mitochondrial mass, meaning that mtDNA copy number was reduced relative to mitochondrial mass in hair cells. This result was not what we had expected. We think the reduced mtDNA copy may have influenced the normal mitochondrial function to a certain extent, giving rise to a high level of ROS and leading to the fragility of hair cells. Therefore, increased mtDNA copy number may have a protective effect on hair cells.

The regulation of intracellular mtDNA copy number is complicated. The catalytic subunit of DNA polymerase $\gamma$ and its processivity factor (both encoded by the POLG2 gene), together with the Twinkle helicase (encoded by the $T W N K$ gene), DNA replication helicase/nuclease 2 (encoded by the $D N A 2$ gene), single-stranded DNA binding protein 1 (encoded by the $S S B P 1$ gene), primase and polymerase (DNA-directed; encoded by the PRIMPOL gene), mitochondrial genome maintenance exonuclease 1 (encoded by the $M G M E 1$ gene), and the mitochondrial transcription factor A (encoded by the $T F A M$ gene), play key roles in mitochondrial DNA maintenance $(13,16,17)$. Of all the genes, TFAM has been the most researched and may be the best choice for increasing the mtDNA copy number. In addition, human TFAM overexpression can increase a mouse cell mtDNA copy number $(9,18)$. Therefore, we used an AAV vector containing the human TFAM gene to increase murine cell mtDNA copy number in our research. After TFAM transfection, we collected the cochlear basal membranes and analyzed the mtDNA by FISH. We found that the mtDNA copy number was increased obviously in transfected hair cells, although the increase lasted just a few days.

In the apoptosis induction experiment, we used neomycin, which is an aminoglycoside antibiotic and can specifically damage hair cells in the cochlear basal membrane but does not damage the surrounding supporting cells (19-21). We added neomycin and found that the hair cells had an obviously lower apoptosis rate after TFAM transfection. The results implied the protective effect of increased mtDNA copy number against apoptosis.

To further confirm the protective effect of mtDNA copy number, we repeated these experiments using HEI-OC1 cells. The results were the same as for hair cells. The mtDNA copy number was increased and remained higher for approximately 1 week after TFAM transfection. During induction of apoptosis, we tried many times but found that the cell line was not sensitive to neomycin. Even at a high concentration of the drug, we found no difference in apoptosis rates between the neomycin-induced and control groups. HEI-OC1 cells originate from mouse cochleas, but they are not real hair cells. Therefore, we finally chose cisplatin. We also found that the TFAM-transfected HEI-OC1 cells had a lower apoptosis rate, consistent with that in hair cells.

Increased mtDNA copy number may exert its protective effect by enhancing the mitochondrial function of the cell. However, the detailed mechanisms are not clear. When the mtDNA copy number was increased in HEI-OC1 cells, we analyzed the transcription of mtDNA genes (NDI, COI, COII, 12SrRNA, ATP6) through PCR, but we found no significant increase (data not shown). Therefore, mitochondrial genes are not activated and involved in the protection against apoptosis.

Mitochondrial DNA is compacted into nucleoids with numerous nucleoid-associated proteins, each nucleoid containing just 1 copy of mtDNA(22-24). As previously reported, 

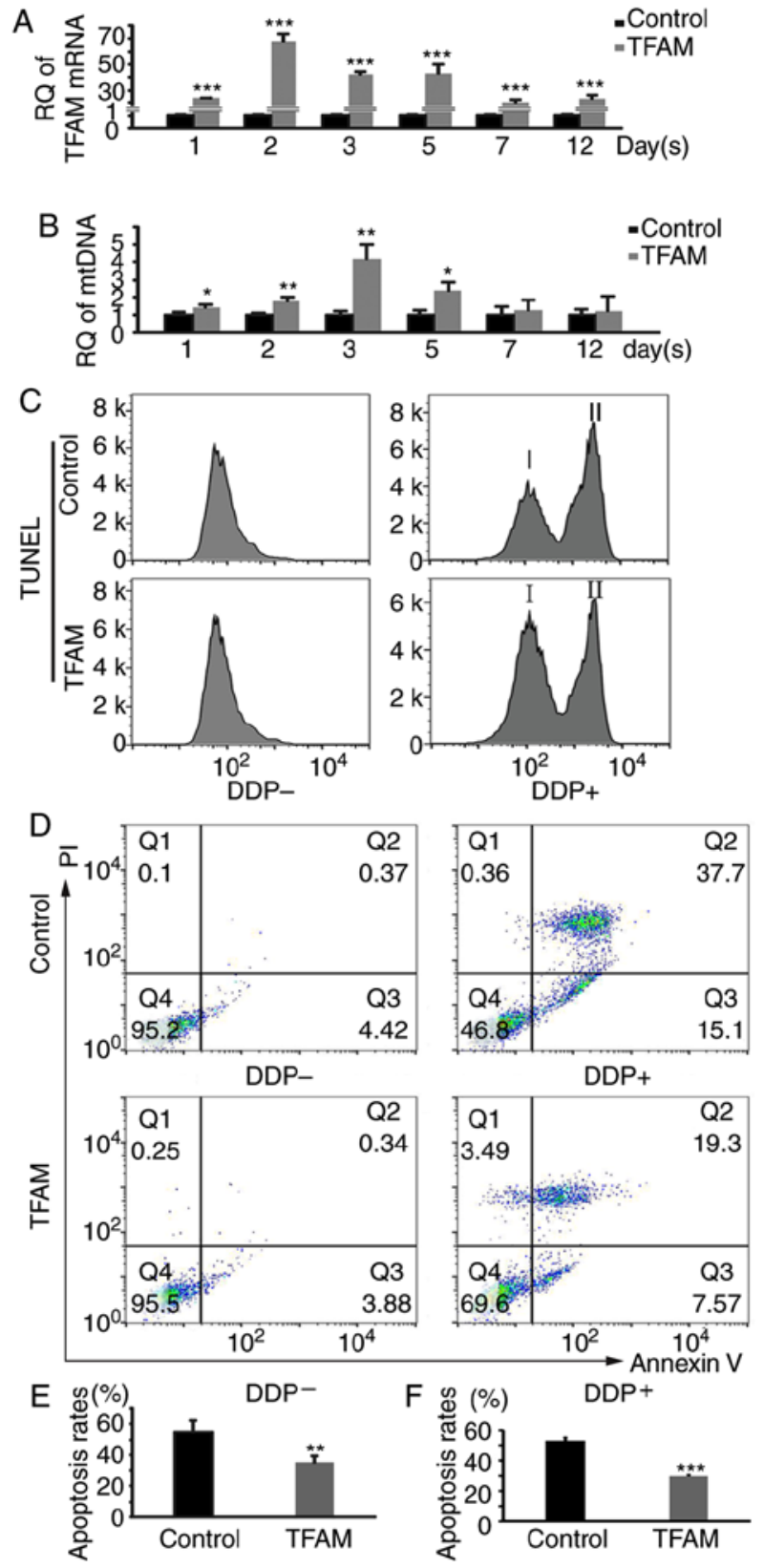

Figure 4. Increased mtDNA copy number has a protective effect on HEI-OC1 cells. (A) Expression levels of TFAM RNA in HEI-OC1 cells. The expression was significantly increased, peaked at the second day $(1 \pm 0.059$; $67.804 \pm 5.377 ; t=21.516 ; \mathrm{n}=3 ; \mathrm{P}=0.0000276$ ) and then decreased. (B) The mtDNA copy number of HEI-OC1 cells as analyzed by qPCR. The number was increased gradually after TFAM transfection, peaked at the third day $(1 \pm 0.199 ; 4.152 \pm 0.808 ; t=6.556 ; \mathrm{n}=3 ; \mathrm{P}=0.0028)$, and then fell back (C) Apoptosis analysis by FCM. We induced apoptosis in HEI-OC1 cells with DDP, and then analyzed the results using TUNEL. I, normal cells; II, apoptotic cells. The apoptosis rate decreased obviously after TFAM transfection. (D) Apoptosis analysis by FCM. We induced apoptosis in HEI-OC1 cells with DDP, and then analyzed the results using Annexin V/PI staining. The apoptosis rate decreased obviously after TFAM transfection (the value of each quadrant was a percentage). (E and F) Quantification of C and D. The results were consistent with that for hair cells $(0.587 \pm 0.035,0.515 \pm 0.013$ $0.46 \pm 0.034,0.274 \pm 0.006 ; \mathrm{t}=4.797,16.067 ; \mathrm{n}=4,3 ; \mathrm{P}=0.00301,0.0000878)$. ${ }^{*} \mathrm{P}<0.05,{ }^{* *} \mathrm{P}<0.01,{ }^{* * *} \mathrm{P}<0.001$. TFAM, mitochondrial transcription factor $\mathrm{A}$; $\mathrm{RQ}$, relatively quantified values; DDP, cisplatin.

the nucleoids attach to the inner membranes of mitochondria and interact with many mitochondrial membrane-bound proteins, such as ATPase family AAA domain-containing

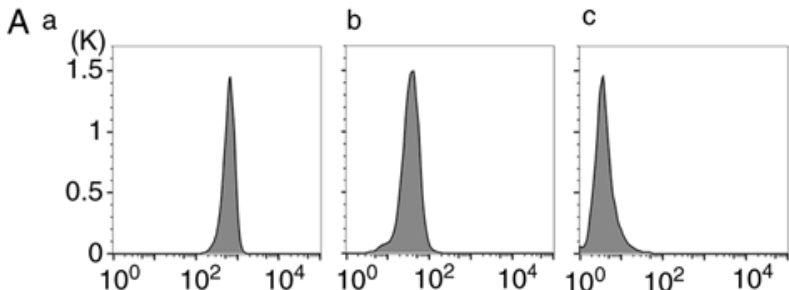

B
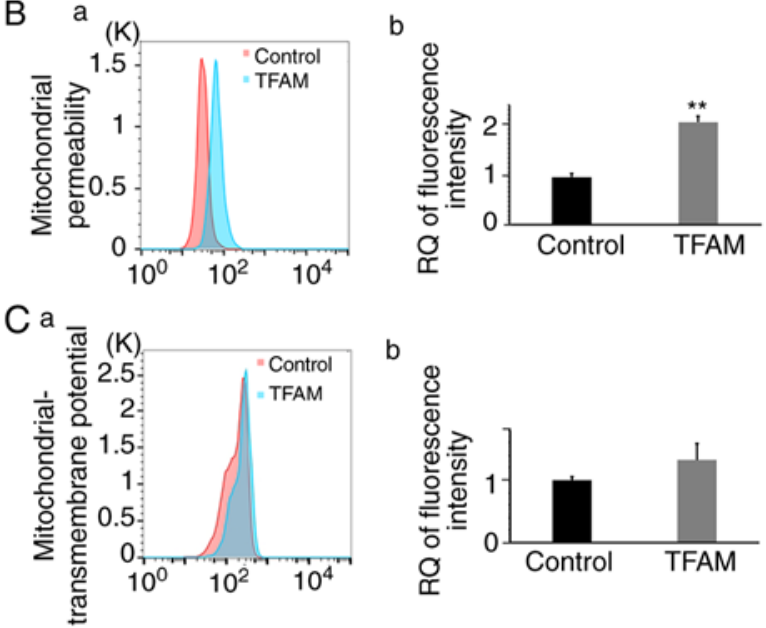

b

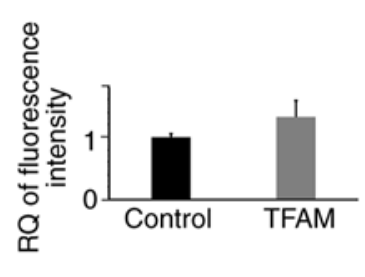

D
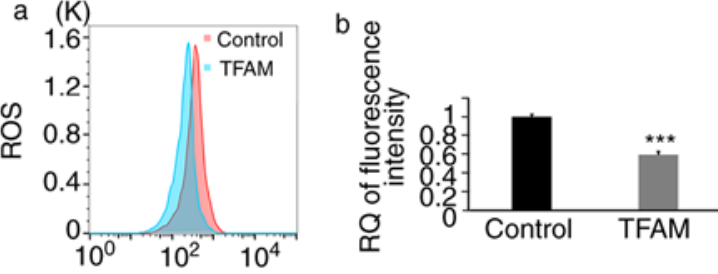

Figure 5. mtDNA copy number affects mitochondrial permeability, MMP, and ROS in HEI-OC1 cells. (A) mPTP assay by FCM; (a) Fluorescent calcein was present in the cytosol as well as the mitochondria, resulting in a bright signal. (b) In the presence of $\mathrm{CoCl}_{2}$, only calcein in the mitochondria emitted a signal and reflected cell mitochondrial permeability; (c) When ionomycin, a calcium ionophore, and $\mathrm{CoCl}_{2}$ were added to the cells at the same time, fluorescence signals were largely abolished. (B) Mitochondrial permeability of HEI-OC1 cells, analyzed by FCM. Fluorescence was obviously stronger in the TFAM group $(1 \pm 0.078 ; 2.144 \pm 0.125 ; t=7.772 ; \mathrm{n}=3 ; \mathrm{P}=0.00148)$. (C) Mitochondrial transmembrane potential analyzed by FCM. This was higher in the TFAM group, but not to a statistically significant degree $(1 \pm 0.053 ; 1.323 \pm 0.264$; $t=2.082, \mathrm{n}=3, \mathrm{P}=0.106$ ). (D) ROS level analyzed by FCM. The level was lower in the TFAM group $(1 \pm 0.024 ; 0.594 \pm 0.034 ; t=9.766 ; \mathrm{n}=3 ; \mathrm{P}=0.000616)$. ${ }^{* * *} \mathrm{P}<0.01,{ }^{* * *} \mathrm{P}<0.001$. MMP, mitochondrial membrane potential; ROS, reactive oxygen species; TFAM, mitochondrial transcription factor A; MPTP, mitochondrial permeability transition pore; $R Q$, relatively quantified values.

protein 3 (ATAD3) $(22,25,26)$. We therefore assume that increased mtDNA copy number may result in a number of mitochondrial nucleoids attaching to the inner membrane, which, together with their interaction with membrane proteins, may further affect mitochondrial-membrane permeability.

The mitochondrial permeability transition pore (mPTP) is formed in the inner membrane of the mitochondrion, and it plays an important role in the physiological regulation of $\mathrm{Ca}^{2+}$ and in ROS homeostasis. The opening of the MPTP initiates the production and release of ROS, which damages mitochondrial and nuclear DNA, proteins, and phospholipids (27-29). In addition, $\mathrm{mPTP}$ has been found to be involved in cell death induced by hypoglycemia, ischemia/reperfusion damage, and neurodegenerative and neuromuscular disorders $(30,31)$. 
We analyzed the mitochondrial permeability of the HEI-OC1 cells by FCM using a mitochondrial-permeability transition pore assay kit and found that permeability was decreased in the TFAM group, which had a higher mtDNA copy number. The results confirmed our hypothesis that the change in the mitochondrial permeability may have influenced the mitochondrial function. We then analyzed the mitochondrial transmembrane potential and the ROS level of the HEI-OC1 cells after TFAM transfection, and found that the potential was higher in the TFAM group (though not to a statistically significant degree), whereas the ROS level was lower. Therefore, decreased mitochondrial permeability enhances the mitochondrial function of cells by stabilizing MMP and reducing ROS, exerting a protective effect on the cells.

In summary, we found that the mtDNA copy number of cochlear hair cells was reduced relative to mitochondrial mass, which may contribute to the fragility of these cells. The mtDNA copy number can be increased in vitro by TFAM overexpression and may help protect the cells against apoptosis. Although the detailed mechanisms, which we intend to study in the future, remain unclear, it is apparent that increased mtDNA copy number may interfere with the opening of the MPTP, reduce the production of ROS and ultimately result in lower rates of apoptosis. These findings provide a potential novel strategy for research into the protection of cochlear hair cells.

\section{Acknowledgements}

Not applicable.

\section{Funding}

The present study was supported by grants from the National Science Foundation for Young Scientists of China (no. 81500788), the National Key R\&D Program of China (nos. 2017YFA0103900, 2016YFC0905200) and the National Natural Science Foundation of China (nos. 81620108005, 81470687, 81830029, 81570913).

\section{Availability of data and materials}

The datasets used and/or analyzed during the current study are available from the corresponding author on reasonable request.

\section{Authors' contributions}

HM, DM and HY performed the experiments, collected the data and wrote the manuscript; SS performed the PCR analysis and collected the data. YC and YZ performed the transfection experiments and collected the data. $\mathrm{RC}$ performed the flow cytometry analysis and collected the data. HL designed the research and gave final approval of the version to be published. HL and SS directed the research and provided supporting materials.

\section{Ethics approval and consent to participate}

Experimental protocols and procedures were reviewed and approved by the Institutional Animal Care and Use Committee of Affiliated Eye and ENT Hospital, Fudan University (Shanghai, China).

\section{Patient consent for publication}

Not applicable.

\section{Competing interests}

The authors declare that they have no competing interests.

\section{References}

1. Zhang W, Kim SM, Wang W, Cai C, Feng Y, Kong W and Lin X: Cochlear gene therapy for sensorineural hearing loss: Current status and major remaining hurdles for translational success. Front Mol Neurosci 11: 221, 2018.

2. Monroe JD, Rajadinakaran G and Smith ME: Sensory hair cell death and regeneration in fishes. Front Cell Neurosci 9: 131, 2015.

3. Atkinson PJ, Huarcaya Najarro E, Sayyid ZN and Cheng AG: Sensory hair cell development and regeneration: Similarities and differences. Development 142: 1561-1571, 2015.

4. Hardie DG: Keeping the home fires burning: AMP-activated protein kinase. J R Soc Interface 15: 20170774, 2018.

5. Cerritelli SM, Frolova EG, Feng C, Grinberg A, Love PE and Crouch RJ: Failure to produce mitochondrial DNA results in embryonic lethality in Rnaseh1 null mice. Mol Cell 11: 807-815, 2003.

6. Mei H, Sun S, Bai Y, Chen Y, Chai R and Li H: Reduced mtDNA copy number increases the sensitivity of tumor cells to chemotherapeutic drugs. Cell Death Dis 6: e1710, 2015.

7. Kalinec GM, Webster P, Lim DJ and Kalinec F: A cochlear cell line as an in vitro system for drug ototoxicity screening. Audiol Neurotol 8: 177-189, 2003.

8. Livak KJ and Schmittgen TD: Analysis of relative gene expression data using real-time quantitative PCR and the 2(-Delta Delta $\mathrm{C}(\mathrm{T})$ ) method. Methods 25: 402-408, 2001.

9. Ekstrand MI, Falkenberg M, Rantanen A, Park CB, Gaspari M, Hultenby K, Rustin P, Gustafsson CM and Larsson NG: Mitochondrial transcription factor A regulates mtDNA copy number in mammals. Hum Mol Genet 13: 935-944, 2004.

10. Goodall-Copestake WP: nrDNA:mtDNA copy number ratios as a comparative metric for evolutionary and conservation genetics. Heredity (Edinb) 121: 105-111, 2018.

11. Hashimoto S, Morimoto N, Yamanaka M, Matsumoto H, Yamochi T, Goto H, Inoue M, Nakaoka Y, Shibahara $\mathrm{H}$ and Morimoto Y: Quantitative and qualitative changes of mitochondria in human preimplantation embryos. J Assist Reprod Genet 34: 573-580, 2017.

12. Birket MJ, Orr AL, Gerencser AA, Madden DT, Vitelli C, Swistowski A, Brand MD and Zeng X: A reduction in ATP demand and mitochondrial activity with neural differentiation of human embryonic stem cells. J Cell Sci 124: 348-358, 2011.

13. Rusecka J, Kaliszewska M, Bartnik E and Tońska K: Nuclear genes involved in mitochondrial diseases caused by instability of mitochondrial DNA. J Appl Genet 59: 43-57, 2018.

14. Foote K, Reinhold J, Yu EPK, Figg NL, Finigan A, Murphy MP and Bennett MR: Restoring mitochondrial DNA copy number preserves mitochondrial function and delays vascular aging in mice. Aging Cell 17: e12773, 2018.

15. Warren EB, Aicher AE, Fessel JP and Konradi C: Mitochondrial DNA depletion by ethidium bromide decreases neuronal mitochondrial creatine kinase: Implications for striatal energy metabolism. PLoS One 12: e0190456, 2017.

16. Gustafsson CM, Falkenberg M and Larsson NG: Maintenance and expression of mammalian mitochondrial DNA. Annu Rev Biochem 85: 133-160, 2016

17. King GA, Hashemi Shabestari M, Taris KH, Pandey AK, Venkatesh S, Thilagavathi J, Singh K, Krishna Koppisetti R, Temiakov D, Roos WH, et al: Acetylation and phosphorylation of human TFAM regulate TFAM-DNA interactions via contrasting mechanisms. Nucleic Acids Res 46: 3633-3642, 2018.

18. Kukat C, Davies KM, Wurm CA, Spåhr H, Bonekamp NA, Kühl I, Joos F, Polosa PL, Park CB, Posse V, et al: Cross-strand binding of TFAM to a single mtDNA molecule forms the mitochondrial nucleoid. Proc Natl Acad Sci USA 112: 11288-11293, 2015. 
19. Lin SCY, Thorne PR, Housley GD and Vlajkovic SM: Resistance to neomycin ototoxicity in the extreme basal (hook) region of the mouse cochlea. Histochem Cell Biol 150: 281-289, 2018.

20. Jiang M, Karasawa T and Steyger PS: Aminoglycoside-induced cochleotoxicity: A review. Front Cell Neurosci 11: 308, 2017.

21. Chen Y, Yu H, Zhang Y, Li W, Lu N, Ni W, He Y, Li J, Sun S, Wang $\mathrm{Z}$ and Li H: Cotransfection of Pax 2 and Math1 promote in situ cochlear hair cell regeneration after neomycin insult. Sci Rep 3: 2996, 2013.

22. Lee SR and Han J: Mitochondrial nucleoid: Shield and switch of the mitochondrial genome. Oxid Med Cell Longev 2017: 8060949, 2017.

23. Gilkerson R, Bravo L, Garcia I, Gaytan N, Herrera A, Maldonado A and Quintanilla B: The mitochondrial nucleoid: Integrating mitochondrial DNA into cellular homeostasis. Cold Spring Harb Perspect Biol 5: a011080, 2013.

24. Kukat C, Wurm CA, Spåhr H, Falkenberg M, Larsson NG and Jakobs S: Super-resolution microscopy reveals that mammalian mitochondrial nucleoids have a uniform size and frequently contain a single copy of mtDNA. Proc Natl Acad Sci USA 108 13534-13539, 2011

25. Gerhold JM, Cansiz-Arda S, Lõhmus M, Engberg O, Reyes A van Rennes H, Sanz A, Holt IJ, Cooper HM and Spelbrink JN: Human mitochondrial DNA-protein complexes attach to a cholesterol-rich membrane structure. Sci Rep 5: 15292, 2015.
26. Desai R, Frazier AE, Durigon R, Patel H, Jones AW, Dalla Rosa I, Lake NJ, Compton AG, Mountford HS, Tucker EJ, et al: ATAD3 gene cluster deletions cause cerebellar dysfunction associated with altered mitochondrial DNA and cholesterol metabolism. Brain 140: 1595-1610, 2017.

27. Rottenberg H and Hoek JB: The path from mitochondrial ROS to aging runs through the mitochondrial permeability transition pore. Aging Cell 16: 943-955, 2017.

28. Treulen F, Uribe P, Boguen R and Villegas JV: Mitochondrial permeability transition increases reactive oxygen species production and induces DNA fragmentation in human spermatozoa. Hum Reprod 30: 767-776, 2015.

29. Zorov DB, Juhaszova M and Sollott SJ: Mitochondrial reactive oxygen species (ROS) and ROS-induced ROS release. Physiol Rev 94: 909-950, 2014.

30. Baines CP and Gutiérrez-Aguilar M: The still uncertain identity of the channel-forming unit(s) of the mitochondrial permeability transition pore. Cell Calcium 73: 121-130, 2018

31. Bernardi P and Di Lisa F: The mitochondrial permeability transition pore: Molecular nature and role as a target in cardioprotection. J Mol Cell Cardiol 78: 100-106, 2015.

This work is licensed under a Creative Commons Attribution-NonCommercial-NoDerivatives 4.0 International (CC BY-NC-ND 4.0) License. 